\title{
FREE EXCITON LUMINESCENCE IN ZnCdSe SOLID SOLUTIONS, ZnSe EPITAXIAL FILMS AND $\mathrm{ZnSe}_{1-x} \mathrm{~S}_{x} / \mathrm{ZnSe}_{1-y} \mathrm{~S}_{y}$ SUPERLATTICES
}

\author{
L.N. Tenishev, S.A. Permogorov, D.L. Fedorov
}

A.F. Ioffe Physical-Technical Institute

Polytechnicheskaya 26, St. Petersburg, 194021, Russia

G.G. YaKushcheva and P.I. Kuznetsov

Institute of Radioengineering and Electronics, Russian Academy of Sciences Vvedensky 1, Fryazino, Moscow Region, 141120, Russia

We studied the luminescence of bulk $\mathrm{Zn}_{1-x} \mathrm{Cd}_{x}$ Se solid solutions, epitaxial ZnSe films and $\mathrm{ZnSe}_{1-x} \mathrm{~S}_{x} / \mathrm{ZnSe}_{1-y} \mathrm{~S}_{y}$ superlattices at $T=2-80 \mathrm{~K}$. The analysis of the bands of exciton radiative annihilation with simultaneous emission of 1 or 2 LO phonons shows that the excitons in the studied samples can be considered as free particles with thermalized distribution of kinetic energies. In $\mathrm{ZnSe}_{1-x} \mathrm{~S}_{x} / \mathrm{ZnSe}_{1-y} \mathrm{~S}_{y}$ superlattices a three-dimensional character of exciton motion was established. The periodic potential of superlattice perturbs the exciton wave vector and induces additional elastic scattering of excitons.

PACS numbers: 78.20 .Ci

\section{Samples}

Bulk samples of cubic $\mathrm{Zn}_{1-x} \mathrm{Cd}_{x}$ Se were grown from vapour phase and had cadmium content $x=0.05-0.07$ as determined from the position of exciton reflection lines. High quality epitaxial $\mathrm{ZnSe}$ films with thickness up to $10 \mu \mathrm{m}$ were grown by photoassisted metal-organic chemical vapour deposition (MOCVD) on (100) GaAs substrates using (Et) $)_{2} \mathrm{Zn},(\mathrm{Met})_{2} \mathrm{Se}$ and $(\mathrm{Et})_{2} \mathrm{~S}$ as sources.

$\mathrm{ZnSe}_{1-x} \mathrm{~S}_{x} / \mathrm{ZnSe}_{1-y} \mathrm{~S}_{y}$ superlattices were grown on $\mathrm{GaAs}$ at $T=450^{\circ} \mathrm{C}$ by photomodulated MOCVD [1]. It was found that the photostimulation with blue light during the growth of $\mathrm{ZnSeS}$ influences the solution composition and hence the band gap of the growing material. By periodic modulation of the photostimulation intensity the superlattices (SL's) consisting of alternating $\mathrm{ZnSeS}$ layers of different composition were grown. Formation of superlattices by this method was confirmed by X-ray analysis. The typical parameters of superlattices were: SL period 5-120 nm; number of periods $-50-300$; total thickness $-2-7 \mu \mathrm{m}$, a verage sulphur content $-2-8 \%$, band-gap modulation $-10-20 \mathrm{meV}$. 


\section{Optical spectra}

All studied samples at helium temperatures demonstrate sharp exciton reflection spectra and bright exciton luminescence. The general character of the exciton emission spectra was similar in all studied samples. As an example, Fig. 1 shows the luminescence spectra of $\mathrm{ZnCdSe}$ bulk crystals at two different temperatures. The most intensive band in the luminescence spectrum closely corresponds in position to the reflection contour of the ground exciton state $(n=1)$ and can be attributed to the zero-phonon emission of excitons. The emission lines of bound excitons $\left(I_{2}\right.$ and $\left.I_{1}\right)$ are much weaker. Besides the resonant exciton line, the luminescence spectra contain the bands $J_{1 \mathrm{LO}}$ and $J_{2 \mathrm{LO}}$, corresponding to the exciton radiative annihilation with simultaneous emission of 1 or 2 LO phonons.

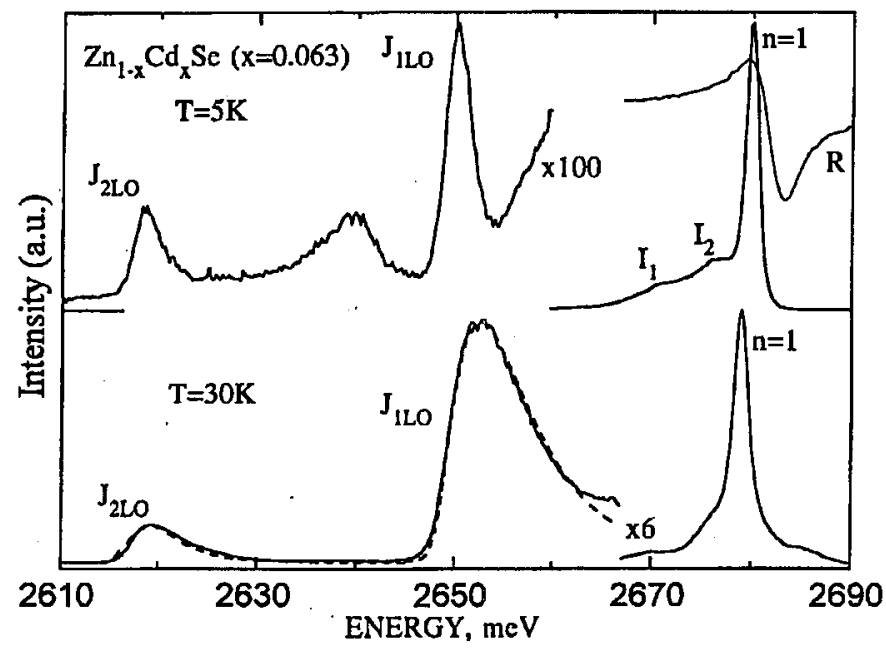

Fig. 1. Exciton luminescence and reflection $(R)$ spectra of bulk $\mathrm{Zn}_{1-x} \mathrm{Cd}_{x} \mathrm{Se}(x=6.3 \%)$ at $T=5$ and $30 \mathrm{~K}$. Dashed line shows a theoretical fit for $J_{1 \mathrm{LO}}$ and $J_{2 \mathrm{LO}}$ phonon assisted bands with Eq. (1) and (2), respectively.

With the increase in temperature the LO asisuled bands broaden and attain a characteristic asymmetric shape. As it is known [2], the shape of these bands reflects the distribution of excitons on kinetic energy. We used the analysis of the band shapes of LO assisted exciton luminescence to study the character of exciton motion and the details of exciton-phonon interaction in our samples.

\section{Theoretical background}

As it follows from momentum conservation, only the excitons with $K=0$ can participate in resonant luminescence. At the same time, in radiative processes with simultaneous emission of phonons the exciton states all over the exciton band with any value of wave vector $\boldsymbol{K}$ can take part. In this case the nonzero exciton momentum is transferred to the emitted phonons. In II-VI polar semiconductors 
the main mechanism of exciton-phonon interaction is the forbidden Frochlich polar scattering of excitons by LO phonons.

In emission this process leads to the appearance of bands with the low-energy thresholds downshifted from the exciton resonance $E_{0}$ by the energy of emitted phonons $E_{0}-N \hbar \omega_{\mathrm{LO}}$. In case of equilibrium exciton distribution in parabolic bands the spectral shapes of 1 and $2 \mathrm{LO}$ assisted bands in accordance with simple perturbation treatment [3] are given by

$$
\begin{aligned}
& J_{1 \mathrm{LO}} \sim \varepsilon^{3 / 2} \exp (-\varepsilon / k T) D_{1 \mathrm{LO}}(\varepsilon), \\
& J_{2 \mathrm{LO}} \sim \varepsilon^{1 / 2} \exp (-\varepsilon / k T) D_{2 \mathrm{LO}}(\varepsilon),
\end{aligned}
$$

where $\varepsilon$ is exciton kinetic energy measured from the corresponding low-energy threshold, $T$ is the bath temperature, $k$ is the Boltzmann constant and $D_{N L O}(N=$ $1,2)$ is the energy denominator of perturbation theory

$$
D_{N L O}=\frac{1}{\left(N \hbar \omega_{\mathrm{LO}}-\varepsilon\right)^{2}+\Gamma^{2}} .
$$

The difference in the shapes of $1 \mathrm{LO}$ and $2 \mathrm{LO}$ bands is caused by the forbidden character of polar Frochlich scattering of excitons by LO phonons. Band shape $J_{2 \mathrm{LO}}$ represents a Maxwellian distribution of exciton kinetic energies. The process with the emission of $1 \mathrm{LO}$ is forbidden and the scattering probability vanishes at $K=0$. As a result, an additional factor reflecting the wave vector dependence of the scattering probability and proportional to the exciton kinetic energy appears in the expression for $J_{1 \mathrm{LO}}$.

For 2 LO process the forbidden character of emission is lifted due to inclusion of intermediate states with alternating parity [3] and the scattering probability does not depend on exciton wave vector and hence its energy.

For the analysis of our data, besides the contour fitting, we had used also the temperature dependence of the band halfwidth $\Delta H_{N L O}$ and the band maximum shift in respect of the low-energy threshold $\Delta M_{N \text { LO }}$. In accordance with Eq. (1) and Eq. (2) these band parameters should linearly increase with temperature as

$$
\begin{aligned}
& \Delta H_{N \mathrm{LO}}=2.95 k T, \quad \Delta H_{N \mathrm{LO}}=1.95 k T, \\
& \Delta M_{N \mathrm{LO}}=(3 / 2) k T, \quad \Delta M_{N \mathrm{LO}}=(1 / 2) k T .
\end{aligned}
$$

The shape of the band with the emission of 1 LO is sensitive to the momentum conservation in luminescence processes. The appearance of elastic scattering breaks the correspondence between the wave vectors of exciton and emitted phonon. As a result, due to the averaging of scattering probability over the phonon wave vectors, the allowed scattering appears in $J_{1 \mathrm{LO}}$ band and its shape can be represented as [4]:

$$
J_{1 \mathrm{LO}}(\varepsilon) \sim\left(\varepsilon+\varepsilon_{0}\right) \varepsilon^{1 / 2} \exp (-\varepsilon / k T),
$$

where the term $\varepsilon_{0}$ represents the contribution of emission processes with perturbation of momentum conservation by elastic scattering. 


\section{Discussion of results}

Figure 2a represents the temperature dependence of halfwidths $\Delta H_{N \mathrm{LO}}$ of $1 \mathrm{LO}$ and $2 \mathrm{LO}$ phonon assisted bands in $\mathrm{ZnCdSe}$ solid solutions. It can be seen that the experimental points are in good agreement with the predictions of Eq. (4). Therefore it can be concluded that the model of equilibrium distribution of free excitons applies well to this case. The dashed line in Fig. 1 shows a fit of the experimental contour of $J_{1 \mathrm{LO}}$ and $J_{2 \mathrm{LO}}$ bands with Eq. (1) and Eq. (2), respectively.
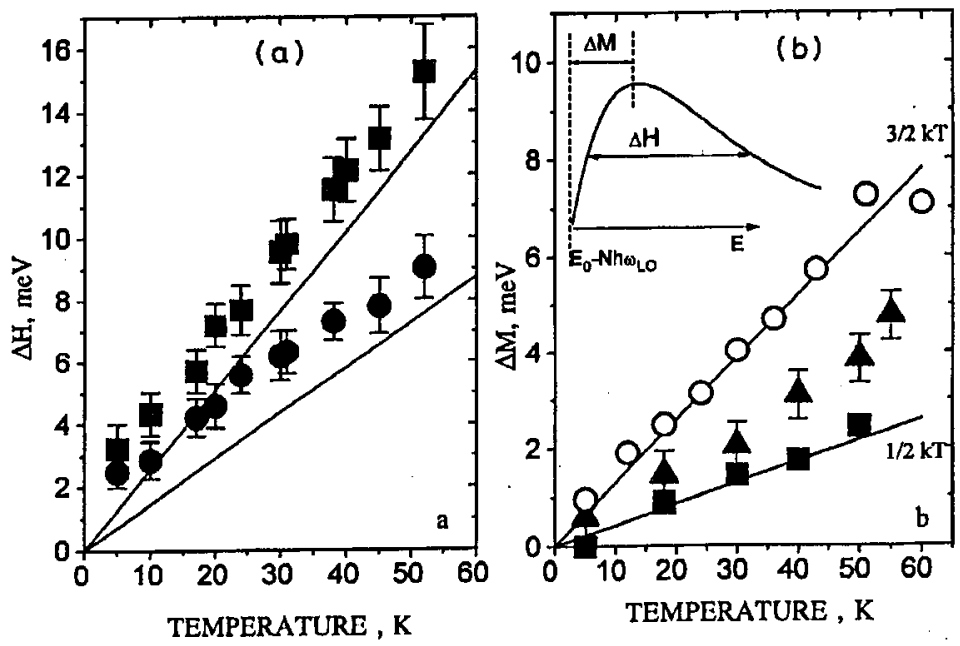

Fig. 2. (a) Temperature dependence of halfwidth $\Delta H_{N L O}$ for $J_{1 L O}$ (squares) and $J_{2 L O}$ (circles) phonon assisted bands in $\mathrm{ZnCdSe}$ sample of Fig. 1. Solid lines represent theoretical predictions. (b) Temperature dependence of band maximum shift $\Delta M_{N L O}$ for $J_{1 \mathrm{LO}}$ (triangles) and $J_{2 \mathrm{LO}}$ (squares) phonon assisted bands in $\mathrm{ZnSe}_{1-x} \mathrm{~S}_{x} / \mathrm{ZnSe}_{1-y} \mathrm{~S}_{y}$ superlattices. Open circles show $\Delta M_{N L O}$ for $\mathrm{ZnSe} / \mathrm{GaAs}$ epilayers.

It should be noted that the observation of free exciton motion in disordered $\mathrm{ZnCdSe}$ solid solutions strongly distinguishes this system with cation substitution from II-VI solid solutions with anion substitution where the strong localization of excitons by composition fluctuations is observed [5].

For ZnSe/GaAs epitaxial films the experimental results are also very well accounted for by the model of phonon-assisted free exciton luminescence with momentum conservation. The equilibrium distribution of excitons in this case indicates the excellent quality of the films.

In case of $\mathrm{ZnSe}_{1-x} \mathrm{~S}_{x} / \mathrm{ZnSe}_{1-y} \mathrm{~S}_{y}$ superlattices we used the shape of $J_{N L O}$ bands to determine the dimensionality of exciton motion. For two-dimensional excitons the density of states should have a step-like character. As a result, in the equilibrium case the maximum of the exciton energy distribution should not shift to higher energies with the increase in temperature [4]. Therefore in $2 D$ case the shift of $J_{2 L O}$ band in respect of the low energy threshold should not change with temperature $\left(\Delta M_{2 \mathrm{LO}}=0\right)$. 
Figure 3 shows reflection $(T=5 \mathrm{~K})$ and emission $(T=5$ and $40 \mathrm{~K})$ spectra of one of the superlattice samples. With the increase in temperature the phonon assisted bands $J_{1 \text { LO }}$ and $J_{2 \text { LO }}$ attain a characteristic shape of exciton kinetic energy distribution which indicates free exciton motion in SL's.

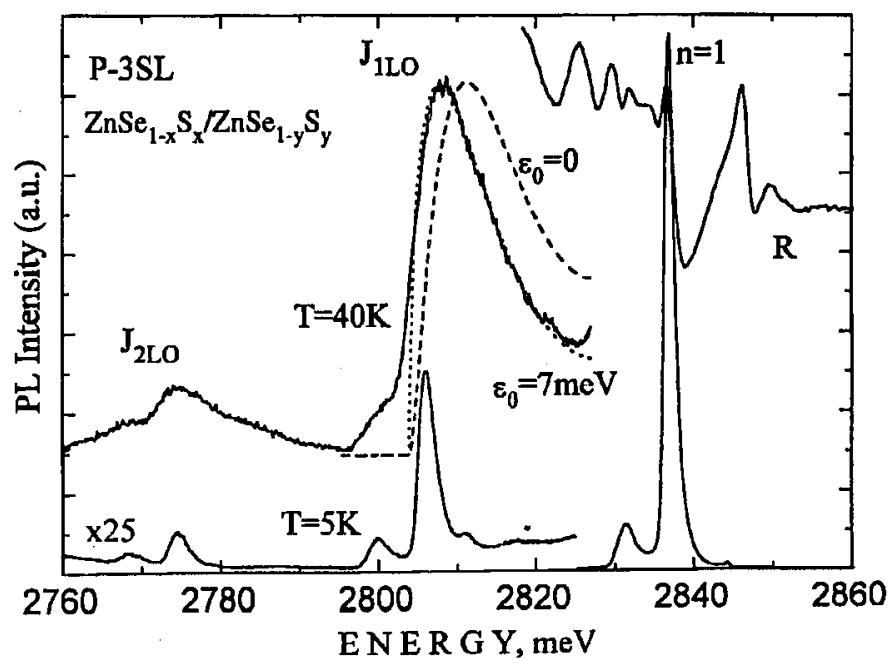

Fig. 3. Exciton luminescence and reflection $(R)$ spectra of $\mathrm{ZnSe}_{1-x} \mathrm{~S}_{x} / \mathrm{ZnSe}_{1-y} \mathrm{~S}_{y}$ superlattices at $T=5$ and $40 \mathrm{~K}$. Dashed lines show a theoretical fit for $J_{1 \mathrm{LO}}$ phonon assisted band with Eq. (6) with $\varepsilon_{0}=0$ and $7 \mathrm{meV}$.

Figure $2 \mathrm{~b}$ shows the temperature dependence of $\Delta M_{N L O}$ in luminescence spectrum of SL. The maxima demonstrate pronounced temperature shift in respect of the threshold positions which unambiguously proves that the exciton motion in our superlattices has a three-dimensional character.

For $\Delta M_{2 \mathrm{LO}}$ a close correspondence to the theoretical prediction of Eq. (5) is observed, whereas the temperature dependence of $\Delta M_{1 L O}$ strongly deviates from theory and is much closer to that for $\Delta M_{2 \mathrm{LO}}$. At the same time the temperature behaviour of $\Delta M_{1 L O}$ in $\mathrm{ZnSe} / \mathrm{GaAs}$ epilayers strictly corresponds to theory. We attributed the modification of band shape $J_{1 \mathrm{LO}}$ in SL's to the effect of elastic scattering of excitons by the periodic potential of superlattice. As it can be seen from Fig. 3 , the band shape of $J_{1 \mathrm{LO}}$, in accordance with this assumption, can be fitted by Eq. (6) with $\varepsilon_{0}=7 \mathrm{meV}$.

\section{Conclusion}

The study of phonon-assisted exciton luminescence provides extensive information on the quality of II-VI epitaxial films and superlattices. The possibility to observe directly the exciton energy distribution gives the information on the character of exciton states (free or localized), the exciton lifetime (longer or shorter than thermalization time), the role of elastic scattering by defects or interfaces and the character of exciton motion (two- or three-dimensional). 


\section{References}

[1] Yu.V. Gulyaev, L.Yu. Zakharov, P.I. Kuznetsov, Thin Solid Films 163, 475 (1988).

[2] S. Permogorov, in: Excitons, Eds. E.I. Rashba, M.D. Sturge, North-Holland, Amsterdam-1982, p. 177.

[3] A.A. Klochikhin, S.A. Permogorov, A.N. Reznitsky, Zh. Eksp. Teor. Fiz. 71, 2230 (1976) [Sov. Phys. JETP 44, 1176 (1976)].

[4] S.A. Permogorov, L.N. Tenishev, A.N. Reznitsky, P.I. Kuznetsov, A.P. Chernushich, L.Yu. Zakharov, G.G. Yakushcheva, J. Cryst. Growth 159, 475 (1996).

[5] S. Permogorov, A. Reznitsky, J. Lumin. 52, 201 (1992). 